\title{
GREEN'S FUNCTIONS OF RANDOM WALKS ON THE UPPER HALF PLANE
}

\author{
KÔHEI UCHIYAMA
}

(Received February 8, 2013)

\begin{abstract}
We obtain an asymptotic estimate of the Green function of a random walk on $Z^{2}$ having zero mean and killed when it exits from the upper half plane. A little more than the second moment condition is assumed. The estimate obtained is used to derive an exact asymptotic form of the hitting distribution of the lower half plane of the walk. The higher dimensional walks are dealt with in the same way.
\end{abstract}

1. Introduction and results. This paper primarily concerns the Green function, denoted by $G_{D}$, of a random walk of zero mean which moves on the two dimensional integer lattice $\boldsymbol{Z}^{2}$ and is killed when it enters into the lower half of the lattice, which we denote by $D$. In [14] an asymptotic form of $G_{D}$ is found in some special cases. In this paper we extend it to a general case under a slightly higher than the second moment condition and apply the result to compute the hitting distribution of $D$. The results obtained are new even if the one step transition is confined in a bounded set; the estimates in them are quite exact, whereas except for some very special case even crude estimates such as upper or lower bounds sharp apart from constant factors are not found in the existing literature to the best of our knowledge.

For any one dimensional recurrent random walk the Green function on a half line has a simple representation by means of a pair of regular and dual-regular functions associated with it and this makes possible to compute its asymptotic form and thereby yields many significant results for the walk [11]. For two dimensional walks we have an analogous representation of $G_{D}$ (see Appendix (B)) but it seems hard to derive directly from it the estimates as obtained in this paper. Tamura and Tanaka [12] find a representation of the Green operator of two dimensional Lévy processes killed on the lower half plane and obtain an explicit form of it for the rotation invariant stable processes. Certain asymptotic estimates of the Green function on the first quadrant are obtained in [2], [9], [10] for the walks of 'nearest neighbour' jumps.

Let $S_{n}^{x}=x+\xi_{1}+\cdots+\xi_{n}$ be a random walk on $Z^{2}$ (the two dimensional integer lattice) starting at $S_{0}^{x}=x \in \boldsymbol{Z}^{2}$. Here $\xi_{j}$ are independent and identically distributed $\boldsymbol{Z}^{2}$-valued random variables defined on a probability space $(\Omega, \mathcal{F}, P)$. It is supposed, throughout this paper but except in the last section, that the walk is irreducible and has zero mean and finite variances. We use complex notation, namely a point $z=\left(z_{1}, z_{2}\right) \in \boldsymbol{R}^{2}$ is denoted by $z_{1}+i z_{2}$, where $i=\sqrt{-1}$, and write $z_{1}=\Re z$ and $z_{2}=\Im$ $z$. Let $D=\left\{z \in Z^{2} ; \Im z \leq 0\right\}$, the lower half

2000 Mathematics Subject Classification. Primary 60G50; Secondary 60J45.

Key words and phrases. Asymptotic formula, Green function, random walk of zero mean and finite variance, hitting probability of half plane. 
of $\boldsymbol{Z}^{2}$, and denote by $G_{D}$ the Green function of the walk killed on $D$ :

$$
G_{D}(x, y)=\sum_{n=0}^{\infty} P_{x}\left[S_{n}=y \quad \text { and } \quad S_{j} \notin D \quad \text { for } j=0,1, \ldots, n\right],
$$

in particular $G_{D}(x, y)=0$ if either $x \in D$ or $y \in D$. Here and throughout the paper $P_{x}$ denotes the probability law of the process $\left(S_{n}^{x}: n=0,1, \ldots\right)$ and $x$ is suppressed from $S_{n}^{x}$ under $P_{x}$. The expectation by $P$ (resp. $P_{x}$ ) is denoted by $E$ (resp. $E_{x}$ ).

Let $X$ and $Y$ be the horizontal and vertical component of $\xi_{1}=S_{1}^{0}$, respectively, put $\sigma_{11}=$ $E X^{2}, \sigma_{12}=E X Y, \sigma_{22}=E Y^{2}, Q=\left(\sigma_{i j}\right)$ (the covariance matrix of $\left.S_{1}^{0}\right)$ and $\sigma=|\operatorname{det} Q|^{1 / 4}$ and define the norm of $z=z_{1}+i z_{2}\left(z_{1}, z_{2} \in \boldsymbol{R}\right)$ by

$$
\|z\|=\sigma^{-1}\left(\sigma_{2}^{2} z_{1}^{2}-2 \sigma_{12} z_{1} z_{2}+\sigma_{1}^{2} z_{2}^{2}\right)^{1 / 2} .
$$

Here (and in what follows) we write $\sigma_{1}^{2}$ and $\sigma_{2}^{2}$ for $\sigma_{11}$ and $\sigma_{22}$, respectively. It is noted that $\|z\|^{2}$ is the quadratic form of the symmetric matrix $\sigma^{2} Q^{-1}$ of determinant 1 ; hence $\|z\|$ agrees with the Euclidean length of $z$ if $Q$ is isotropic. For $a, b \in \boldsymbol{R}, a \vee b$ and $a \wedge b$ denote the maximum and minimum, respectively, of $a$ and $b$. The function $t \log t$ is understood continuously extended to $t=0$.

The vertical component process $\Im S_{n}$ is a random walk on $\boldsymbol{Z}$ that is irreducible, recurrent and of finite variance. Let $f_{+}(n)$ (resp. $\left.f_{-}(n)\right)(n=1,2, \ldots)$ be the positive function on $\{n>0\}$ that is asymptotic to $n$ and regular with respect to $\Im S_{n}$ (resp. $-\Im S_{n}$ ) killed on $\{n \leq 0\}$ :

$$
f_{ \pm}(n)=E\left[f_{ \pm}(n \pm Y) ; n \pm Y>0\right](n \geq 1) \text { and } \lim _{n \rightarrow \infty} f_{ \pm}(n) / n=1,
$$

which exist each uniquely (Spitzer [11, P19.5]) (Proposition 5 of Section 19) and [11, E27.3]). (It is $[0, \infty)$ rather than $[1, \infty)$ that the regular functions are considered in [11], so that $f_{+}=$ $A_{+} f(\cdot-1)$ and $f_{-}=A_{-} g(\cdot-1)$ with $f, g$ in $[11, \mathrm{P} 19.5]$ and certain positive constants $A_{ \pm}$; see $[11, \mathrm{P} 18.8]$ for the latter.) Put

$$
\tilde{X}=X-\left(\sigma_{12} / \sigma_{2}^{2}\right) Y .
$$

THEOREM 1. Suppose that $E\left[\tilde{X}^{2} \log |\tilde{X}|\right]<\infty$. Then for $n>0, k>0$,

$$
G_{D}(i n, s+i k)=\frac{2 f_{+}(n) f_{-}(k)}{\pi \sigma_{2}^{2}\|s+i(k-n)\|^{2}}(1+o(1)) \quad \text { as } \frac{n k}{\|s+i(k-n)\|^{2}} \rightarrow 0 .
$$

In the special case when the walk is mirror-symmetric relative to the real axis and the vertical component is continuous (i.e., $P[|Y| \geq 2]=0$ ), the reflection principle is available and the known asymptotic form of the potential function of the walk on the whole lattice $\boldsymbol{Z}^{2}$ ([6], [11]) immediately gives the formula of Theorem 1 with $f_{ \pm}(n)=n$ (at least under the higher moment condition). When the vertical component is left or right continuous, the formula above is obtained in [14] (see Remark 7 below for more details).

REMARK 1. In the case when $k n /\|s+i(k-n)\|^{2}$ is bounded away from zero we can obtain some estimates of $G_{D}$ from [14, Theorem 1.1]. It follows that for any $\varepsilon>0$ there 
exists a number $C$ such that if $k n /\|s+i(k-n)\|^{2}>\varepsilon$, then

$$
G_{D}(i n, s+i k) \leq C \log \left(1+\frac{n k}{\|s+i(k-n)\|^{2} \vee 1}\right) .
$$

Under a little stronger moment condition we have a rather precise estimate: if the moment condition $E\left[\left|S_{1}^{0}\right|^{2} \log \left|S_{1}^{0}\right|\right]<\infty$ is vaild and $G_{D}$ is put in the form

$$
G_{D}(i n, s+i k)=\frac{1}{2 \pi \sigma^{2}} \log \left(1+\frac{4\left(\sigma / \sigma_{2}\right)^{2} n k}{\|s+i(k-n)\|^{2} \vee 1}\right)+B_{s, n, k}
$$

( $n>0, k>0, s \in \boldsymbol{Z}$ ), then $B_{s, n, k}$ converges to zero as both $k \wedge n$ and $\|s+i(k-n)\|$ tend to infinity, and is uniformly bounded (see Lemma 7 in Section 3).

REMARK 2. The function $h(z)=f_{+}(\Im z)$ is regular on $Z^{2} \backslash D$ in the sense that $h \geq 0$ and $h(z)=E_{z}\left[h\left(S_{1}\right) ; S_{1} \notin D\right]$. From Theorem 1 it follows that the positive multiples of it are the only positive regular functions on $Z^{2} \backslash D$, thus determining the Martin boundary of the killed walk, provided that $E\left[\tilde{X}^{2} \log |\tilde{X}|\right]<\infty$ (cf. [11, P27.3]. This moment condition should be relaxed, although the present author does not know how it could be done. It however holds that if $\Im S_{n}$ is recurrent (but no moment condition is required), then $f_{+}(\Im z)$ is minimal, namely any regular function on $Z^{2} \backslash D$ that is dominated by $f_{+}(\Im z)$ must be a positive multiple of $f_{+}(\Im z)$. We shall prove this fact in the last section (Theorem 11) by using the method of Choquet and Deny [4]. Martin boundary for the random walks on a half space or a quadrant are recently investigated by several authors (see [7], [10] and the references contained in them).

REMARK 3. The condition $E\left[\tilde{X}^{2} \log |\tilde{X}|\right]<\infty$ cannot be removed for the estimate of Theorem 1 (see [15, Theorem 3], also [13]), whereas it would be superfluous (under our basic framework) for the asymptotic formula of $\sum_{t \geq s} G_{D}(i n, t+i k)$.

Let $H_{D}(x, y)$ denote the probability that the walk starting at $x$ hits $D$ for the first time at $y \in D: H_{D}(x, y)=P\left[S_{\tau(D)}^{x}=y\right]$, where $\tau(D)=\inf \left\{j>0 ; S_{j}^{x} \in D\right\}$. Then for $n>0, k \leq 0$ (and $s \in \boldsymbol{Z}$ ),

$$
H_{D}(i n, s+i k)=E\left[G_{D}(i n, s-X+i(k-Y)) ; Y<k\right] .
$$

Put

$$
q(k)=\frac{2}{\sigma_{2}^{2}} E\left[f_{-}(k-Y) ; Y<k\right] \quad(k \leq 0) .
$$

Using Theorem 1 we shall derive the following result.

THEOREM 2. Suppose that $E\left[\left|S_{1}^{0}\right|^{2} \log \left|S_{1}^{0}\right|\right]<\infty$. Then for $n>0$ and $k \leq 0$, as $|s| \vee(n-k) \rightarrow \infty$

$$
H_{D}(i n, s+i k)=\frac{1}{\pi} \frac{f_{+}(n) q(k)}{\|s+i(k-n)\|^{2}}\left(1+o_{b}(1)\right)+r(n, s, k)
$$

where $o_{b}(1)$ is bounded and approaches 0 as far as $k$ remains in any bounded set,

$$
r(n, s, k)=O\left(\log (n+1) / M^{2} \log M\right) \quad \text { with } \quad M=\|s+i(k-n)\|,
$$


and as $|s| \vee n \rightarrow \infty$

$$
\sum_{k \leq 0} r(n, s, k)=o\left(\frac{n}{s^{2}+n^{2}}\right) .
$$

Theorem 2 has the following corollary.

COROLlary 3. Suppose that $E\left[\left|S_{1}^{0}\right|^{2} \log \left|S_{1}^{0}\right|\right]<\infty$. Then for $n>0$, as $|s| \vee n \rightarrow \infty$

$$
\sum_{k \leq 0} H_{D}(i n, s+i k)=\frac{1}{\pi} \frac{f_{+}(n)}{\|s-i n\|^{2}}(1+o(1)) \text {. }
$$

REMARK 4. The function $q$ given in (4) is a probability law on $\{0,-1,-2, \ldots\}$. It is the limit of the law of $\Im S_{\tau(D)}^{i n}$ as $n \rightarrow \infty$ :

$$
q(k)=\lim _{n \rightarrow \infty} P_{i n}\left[\Im S_{\tau(D)}=k\right] \quad(k \leq 0)
$$

(cf. [11, P19.4, P18.8]). The bound of $r$ in (6), not enough to yield (7), is crude. Better bounds are obtained from the estimates (34) and (37) given in its proof, especially when the existence of higher moments are assumed. The error term $o_{b}(1)$ may remain away from zero along a subsequence. To have $\lim o_{b}(1)=0$ we need some regular behavior of the tail $P[Y<k]$ as $k \rightarrow-\infty$.

REMARK 5. The determinant of the quadratic form $\|\cdot\|^{2}$ is unity as noted previously. Hence

$$
\lim _{n \rightarrow \infty} \sum_{s \in \boldsymbol{Z}} \frac{n}{\pi\|s+i(k-n)\|^{2}}=\int_{\boldsymbol{R}} \frac{|u|}{\pi\|t-i u\|^{2}} d t=1 \quad(u \in \boldsymbol{R} \backslash\{0\}) .
$$

By the same token the invariance principle gives that $\sum_{s \leq x} \sum_{k \leq 0} H_{D}(i n, s+i k)$ approaches $\pi^{-1} \int_{-\infty}^{\xi}\|t-i\|^{-2} d t$ as $s / n \rightarrow \xi$ for each $\xi \in \boldsymbol{R}$. These of course conform to (8).

REMARK 6. Denote by $S_{n}^{*}$ the $h$-process of the killed walk with the regular function $h(z)=f_{+}(\Im z)$. Its $n$ step transition law $p_{n}^{*}$ is given by

$$
p_{n}^{*}(x, y)=P_{x}\left[S_{n}=y, \tau(D)>n\right] f_{+}(\Im y) / f_{+}(\Im x), \quad x, y \notin D ;
$$

its Green function $G_{D}^{*}\left(z_{0}, z\right)$ satisfies

$$
G_{D}^{*}(i n, s+i k)=\frac{2 f_{+}(k) f_{-}(k)}{\pi \sigma_{2}^{2}\|s+i(k-n)\|^{2}}(1+o(1)) \quad \text { as } \frac{n k}{\|s+i(k-n)\|^{2}} \rightarrow 0
$$

owing to Theorem 1 . The functional limit theorem holds for the process $X_{n}^{*}(t):=n^{-1 / 2} S_{[t n]}^{*}$, $t \geq 0$, the limit process is the $h$-transform with $h(z)=\Im z$ of the Brownian motion of mean zero and covariance matrix $Q$ killed when it hits the real line; its Green function $g\left(\zeta_{0}, \zeta\right)$ is identified as $2(\Im \zeta)^{2} / \pi \sigma_{2}^{2}\left\|\zeta-\zeta_{0}\right\|^{2}(\zeta, \zeta \in C \backslash D)$. (The proof can be readily reduced to one dimensional problem of showing the convergence of the vertical component, which is established in the classical paper by Pitman [8] if $P[Y= \pm 1]=1 / 2$ and solved in [3] for walks attracted to stable processes.) The formula (11) provides a close approximation for the Green function especially in the interesting case when $\Im X_{n}^{*}(0) \rightarrow 0$. 
Let $L=\left\{z \in Z^{2} ; \Im z=0\right\}$, the real axis, and denote by $G_{L}$ the Green function on $Z^{2} \backslash L$ :

$$
G_{L}(x, y)=\sum_{n=0}^{\infty} P_{x}\left[S_{n}=y, S_{j} \notin L \text { for } j=1, \ldots, n\right] .
$$

Let $a(n), n \in \boldsymbol{Z}$, denote the potential function of the one dimensional walk $\mathfrak{\Im} S_{n}$ :

$$
a(n)=\sum_{j=0}^{\infty}\left(P_{0}\left[\Im S_{j}=0\right]-P_{0}\left[\Im S_{j}=-n\right]\right) .
$$

It holds that $a(n)=\sigma_{2}^{-2}|n|+o(n)$ as $|n| \rightarrow \infty$. (cf. [11, T28.1, P29.2].) The proof of Theorem 1 depends on the next theorem established in [14].

Theorem A. Suppose that $E\left[\tilde{X}^{2} \log |\tilde{X}|\right]<\infty$. Then it holds that for $n>0, k \in \boldsymbol{Z}$, as $n(k \vee 1) /\|s+i(k-n)\|^{2} \rightarrow 0$,

$$
G_{L}(i n, s+i k)=\frac{1}{\pi} \frac{\sigma_{2}^{4} a(n) a(-k)+n k}{\sigma_{2}^{2}\|s+i(k-n)\|^{2}}+o\left(\frac{n k}{\|s+i(k-n)\|^{2}}\right) .
$$

We have a nice trigonometric representation of $G_{L}$ and the proof of Theorem A is carried out by direct computation based on it. Although no such one is available for $G_{D}$, by writing down the difference $G_{L}-G_{D}$ by means of $G_{L}$ and $H_{D}$ we can deduce from Theorem A an intermediate estimate of $G_{D}$, which is eventually sharpened by some probabilistic argument to the estimate of Theorem 1. We have a similar (but much simpler) situation for the transition probability of one dimensional walk killed on a negative half line (cf.[17]). For the present problem we need to control the deviation of the walk $S_{n}$ not only in the vertical but also in the horizontal direction and to this end we shall verify Lemma 6 of the next section.

REMARK 7. Suppose the one-dimensional walk $\Im S_{n}$ to be right-continuous, namely $P[Y \geq 2]=0$. Then (i) $G_{D}$ agrees with $G_{L}$ restricted on the upper half plane and (ii) for $n>0$,

$$
\sigma_{2}^{2} a(-n)=n, f_{+}(n)=\left(\sigma_{2}^{2} a(n)+n\right) / 2 \text { and } f_{-}(n)=n,
$$

(cf. [11, P30.3]), hence the formula of Theorem 1 follows from that of Theorem A, which is of course also the case if $\Im S_{n}$ is left continuous.

Theorem 1 in turn is used to improve the estimate of $G_{L}$ in the case $n k<0$ under a stronger moment condition. If $E|Y|^{3}<\infty$, put

$$
\lambda_{3}=\frac{1}{3 \sigma_{2}^{2}} E\left[Y^{3}\right] \quad \text { and } \quad C^{*}=\frac{1}{2 \pi} \int_{-\pi}^{\pi}\left[\frac{\sigma_{2}^{2}}{1-E\left[e^{i t Y}\right]}-\frac{1}{1-\cos t}\right] d t .
$$

THEOREM 4. Suppose that $E\left[\left|S_{1}^{0}\right|^{3} \log (|Y| \vee 2)\right]<\infty$. Then, uniformly in $s$, as $|n| \wedge$ $|k| \rightarrow \infty$ under the constraint $n k<0$,

$$
G_{L}(i n, s+i k)=\left(C^{*}-\lambda_{3} \operatorname{sgn} n\right) \frac{|k-n|}{\pi \sigma_{2}^{2}\|s+i(k-n)\|^{2}}(1+o(1)),
$$

where $\operatorname{sgn} n=n /|n|(n \neq 0)$. 
REMARK 8. Suppose $E|Y|^{3}<\infty$. Then it holds ([17, Appendix A]) that

(i) $\sigma_{2}^{2} a(n)-|n|=C^{*}-\lambda_{3} \operatorname{sgn} n+o(1)$ as $|n| \rightarrow \infty$;

(ii) $C^{*}-\lambda_{3}=\sum_{k<0} q(k)\left(\sigma_{2}^{2} a(k)+|k|\right)$ and $C^{*}+\lambda_{3}=\sum_{k>0} q^{-}(k)\left(\sigma_{2}^{2} a(k)+k\right)$, where $q^{-}(k)=\frac{2}{\sigma_{2}^{2}} E\left[f_{+}(Y-k) ; Y>k\right]$ for $k \geq 0$; in particular

(iii) $C^{*}-\lambda_{3}>0$ (resp. $C^{*}+\lambda_{3}>0$ ) unless the walk is left continuous (resp. right continuous).

Theorem 4 says, in view of (i) above, that in the formula of Theorem A the numerator $k n$ of the error term can be replaced by $|n| \vee|k|$ for $n k<0$, provided that the moment condition is suitably strengthened.

All the results are readily extended to the dimensions $d \geq 3$ without any essential change in the derivation. We state only the extention of Theorem 1 which may read as in the next theorem. Let $S_{n}^{x}=x+\xi_{1}+\cdots+\xi_{n}$ on $\boldsymbol{Z}^{d}$ be a random walk on $\boldsymbol{Z}^{d}$. Denote by $Y$ the projection of $\xi_{1}$ to the $d$-th axis and by $X$ that to the hyperplane perpendicular to it so that $\xi_{1}=$ $(X, Y)$. Let $G_{D}$ the Green function analogously defined with $D=\left\{s+i k ; s \in Z^{d-1}, k=\right.$ $0,-1,-2, \ldots\}$, where $s+i k$ stands for the $d$-dimensional point $\left(s_{1}, \ldots, s_{d-1}, k\right) \in Z^{d}$.

THEOREM 5. Suppose that the walk $S_{n}^{x}$ on $Z^{d}$ is irreducible and $E\left[\xi_{1}\right]=0$ and that

$$
E\left[|X|^{d}\right]<\infty(d \geq 3) ; \quad E\left[|Y|^{2} \log |Y|\right]<\infty(d=3), \quad E\left[|Y|^{d-1}\right]<\infty(d \geq 4) .
$$

Then for $n>0, k>0$ and $s \in Z^{d-1}$, as $n k /\|s+i(k-n)\|^{2} \rightarrow 0$,

$$
G_{D}(i n, s+i k)=\frac{\Gamma(d / 2)}{\pi^{d / 2}} \cdot \frac{2 f_{+}(n) f_{-}(k)}{\sigma_{d}^{2}\|s+i(k-n)\|^{d}}(1+o(1)) .
$$

Here $\sigma_{d}^{2}$ denotes the variance of the $d$-th component of $S_{1}^{0}$ and the norm $\|\cdot\|$ is defined analogously to the two dimensional case.

In Section 2 we prove Lemma 6 as previously mentioned. The proofs of Theorems 1, 2 and 4 are given in Sections 3, 4 and 5, respectively.

2. A preliminary lemma. Here we prove the next lemma that plays a crucial role in the proof of Theorem 1; for it we need only our basic moment conditions $E_{0}\left|S_{1}\right|^{2}<\infty$ and $E_{0} S_{1}=0$. For $A \subset Z^{2}$ let $\tau(A)=\inf \left\{n \geq 1 ; S_{n} \in A\right\}$ as in the definition of $H_{D}(x, y)$. Sometimes $\tau_{A}$ is written for $\tau(A)$.

Lemma 6. For $N, M>1$, let $A=A(N, M)=\left\{x+i y \in Z^{2} ; x \geq-M, 1 \leq y \leq N\right\}$. Then as $M / N \rightarrow \infty$

$$
\sup _{1 \leq n \leq N} P_{i n}\left[\Re S_{\tau\left(Z^{2} \backslash A(N, M)\right)}<-M\right]=o\left((N / M)^{2}\right) .
$$

PROOF. It suffices to consider

$$
B(N)=A(2 N, M)+M-i N=\left\{z \in Z^{2} ; x \geq 0,-N \leq y \leq N\right\}
$$


instead of $A(N, M)$ and prove

$$
\sup _{|n| \leq N} P_{M+i n}\left[\Re S_{\tau\left(Z^{2} \backslash B(N)\right)}<0\right]=o\left((N / M)^{2}\right) .
$$

The proof rests on the maximum principle that is described below. Put

$$
h_{N}(z)=P_{z}\left[\Re S_{\sigma(B(N))}<0\right] \quad \text { where } \sigma(B(N))=\inf \left\{n \geq 0 ; S_{n} \notin B(N)\right\} .
$$

Then if a function $\varphi_{N}(z), z \in Z^{2}$ is bounded below and superharmonic on $B(N)$, namely

$$
\mathrm{P} \varphi_{N}(z):=E\left[\varphi_{N}(z+X+i Y)\right] \leq \varphi_{N}(z) \text { for } z \in B(N),
$$

and satisfies the boundary condition

$$
\varphi_{N}(x+i y) \geq 1 \text { if } x<0 \quad \text { and } \quad \varphi_{N}(x+i y) \geq 0 \quad \text { if }|y| \geq N,
$$

we can conclude that $\varphi_{N} \geq h_{N}$ on $B(N)$. In fact, $h_{N}$ is harmonic on $B(N)$ and taking values 1 or 0 according as $x<0$ or $x \geq 0$ on the boundary $Z^{2} \backslash B(N)$, and from the condition imposed on $\varphi_{N}$ it therefore follows that $f_{N}:=\varphi_{N}-h_{N}$ is superharmonic on $B(N)$ and non-negative on $\boldsymbol{Z}^{2} \backslash B(N)$, so that $f_{N}(z) \geq E_{z}\left[f_{N}\left(S_{\tau\left(\boldsymbol{Z}^{2} \backslash B(N)\right)}\right)\right] \geq 0$.

In the rest of this proof we construct $\varphi_{N}$ which satisfies for every sufficiently large $N$ not only the properties described above but also the condition

$$
\sup _{|y| \leq N} \varphi_{N}(x+i y)=o\left((N / x)^{2}\right) \quad \text { as } x / N \rightarrow \infty,
$$

which is enough to conclude (12). The construction is carried out in three steps.

Step 1. From our moment condition on $X$ it follows that there exists a smooth positive function $u(s), s \geq 0$ such that

$$
u(0)=0, \quad u^{\prime} \geq 0, \quad \sup |u(2 s)-u(s)|<\infty
$$

and as $s \rightarrow \infty$

$$
s^{2} e^{u(s)} P[X<-s] \rightarrow 0
$$

and

$$
u(s) \rightarrow \infty, \quad u^{\prime}(s) \rightarrow 0, \quad u^{\prime \prime}(s) \rightarrow 0 .
$$

Let $h(s)$ be a smooth function on $\boldsymbol{R}$ such that

$$
\begin{aligned}
& h(s)=(1+s)^{-2} e^{-u(s)} \quad \text { if } s>0 ; \\
& 1=h(0) \leq h(s)<2 \quad \text { and } \quad h^{\prime}(s) \leq 0 \text { if } s \leq 0 .
\end{aligned}
$$

From (17) it follows that

$$
h^{\prime}(s) / h(s) \rightarrow 0 \quad \text { and } \quad h^{\prime \prime}(s) / h(s) \rightarrow 0 \text { as } s \rightarrow \infty .
$$

We further bring in a function $\varphi(s, t)$ on $\boldsymbol{R}^{2}$ given by

$$
\varphi(s, t)= \begin{cases}C_{0} h\left(s+s_{0}\right) \cos (\pi t / 4) & \text { if }|t| \leq 4 / 3 \\ C_{0} h\left(s+s_{0}\right) \lambda(|t|) & \text { if }|t|>4 / 3\end{cases}
$$


Here $\lambda(t), t>0$ is a smooth function with $\lambda^{\prime} \leq 0, \inf \lambda(t)>0$ and chosen so that the partial derivatives $\partial_{t} \varphi$ and $\partial_{t}^{2} \varphi$ exist and continuous; the constants $s_{0}>0$ and $C_{0}>0$ are chosen, in turn, so that

$$
\begin{aligned}
\sup _{|t|<1} \frac{\sigma_{1}^{2}\left|\varphi_{s s}(s, t)\right|+2 \overline{\sigma_{12}}\left|\varphi_{s t}(s, t)\right|}{\varphi(s, t)} & =\frac{\sigma_{1}^{2}\left|h^{\prime \prime}\left(s+s_{0}\right)\right|}{h\left(s+s_{0}\right)}+\frac{2 \pi \overline{\sigma_{12}}\left|h^{\prime}\left(s+s_{0}\right)\right|}{\sqrt{3} h\left(s+s_{0}\right)} \\
& \leq \sigma_{2}^{2}\left[(\pi / 4)^{2}-1 / 4\right] \quad \text { for } s>-1,
\end{aligned}
$$

where $\varphi_{t}=\partial_{t} \varphi, \varphi_{t s}=\partial_{s} \partial_{t} \varphi$ etc. and $\overline{\sigma_{12}}=E[|X Y|]$, and then that $C_{0} h\left(s_{0}\right) \inf \lambda(t) \geq 1$. (The constant appearing as the upper bound in (18) may be an arbitrary positive constant, so that the supremum above can be made arbitrarily small, which fact though not applied connotes that $\varphi_{s t}$ and $\varphi_{s s}$ are intrinsically negligible in comparison to $\varphi_{t t}$; one given in this form is only for convenience sake.) It then follows that

$$
\begin{gathered}
\Delta_{Q} \varphi \leq-\frac{1}{4} \sigma_{2}^{2} \varphi(s \geq 0,|t| \leq 1), \\
\varphi \geq 1 \quad(s \leq 0), \quad \varphi \geq 0 \text { and } \sup _{t} \varphi(s, t)=o\left(s^{-2}\right)(s \rightarrow \infty),
\end{gathered}
$$

where $\Delta_{Q}=\sigma_{1}^{2} \partial_{s}^{2}+2 \sigma_{12} \partial_{t} \partial_{s}+\sigma_{2}^{2} \partial_{t}^{2}$.

Step 2. Now define

$$
\varphi_{N}(z)=\varphi(x / N, y / N) \quad\left(z=x+i y \in Z^{2}\right) .
$$

Clearly the conditions (13) and (14) are satisfied. It is easy to see that as $(x / N, y / N) \rightarrow(s, t)$

$$
\lim _{N \rightarrow \infty} \frac{N^{2}(\mathrm{P}-1) \varphi_{N}(z)}{\varphi_{N}(z)}=\frac{\frac{1}{2} \Delta_{Q} \varphi(s, t)}{\varphi(s, t)}<-\frac{\sigma_{2}^{2}}{8} \quad \text { for } s \geq 0,|t| \leq 1 .
$$

In order to conclude from this relation that the inequality $\mathrm{P} \varphi_{N} \leq \varphi_{N}$ holds on $B(N)$ (for all sufficiently large $N)$ we need some uniformity in $(s, t)$ of this convergence or something alternative for it. Here we simply claim that for all $N$ large enough the ratio under the limit is less than $-\sigma_{2}^{2} / 16$ on $B(N)$, which certainly assures the desired inequality, hence (12).

Looking at

$$
N^{2}(\mathrm{P}-1) \varphi_{N}(z)=N^{2} E\left[\varphi\left(\frac{x+X}{N}, \frac{y+Y}{N}\right)-\varphi\left(\frac{x}{N}, \frac{y}{N}\right)\right],
$$

we simultaneously add and subtract the term $\varphi\left(\frac{x}{N}, \frac{y+Y}{N}\right)$ inside the expectation symbol and accordingly break the right side into two parts of which the second one divided by $\varphi_{N}(z)$ is convergent:

$$
\frac{N^{2} E\left[\varphi\left(\frac{x}{N}, \frac{y+Y}{N}\right)-\varphi\left(\frac{x}{N}, \frac{y}{N}\right)\right]}{\varphi_{N}(z)} \rightarrow \frac{\sigma_{2}^{2}}{2} \cdot \frac{\varphi_{t t}(s, t)}{\varphi(s, t)}=-\frac{1}{2}[\pi / 4]^{2} \sigma_{2}^{2} .
$$

Here the convergence is certainly uniform in $(s, t)$. The other one, which we call $K_{N}$, is $K_{N}=$ $N^{2} E\left[\varphi\left(\frac{x+X}{N}, \frac{y+Y}{N}\right)-\varphi\left(\frac{x}{N}, \frac{y+Y}{N}\right)\right]$. As for this expectation, evaluation of the contribution of 
large negative values of $X$ is somewhat troublesome. To dispose of it we obtain that for each $\varepsilon>0$, as $N \rightarrow \infty$

$$
\frac{N^{2}}{\varphi_{N}(z)} E\left[\left|\varphi\left(\frac{x+X}{N}, \frac{y+Y}{N}\right)-\varphi\left(\frac{x}{N}, \frac{y+Y}{N}\right)\right| ; X<-\varepsilon N\right] \rightarrow 0
$$

uniformly in $(x, y)$, of which the proof is postponed to the last step.

Applying, successively, Taylor's expansion to $\varphi\left(\cdot, \frac{y+Y}{N}\right)$ and the mean value theorem to $\partial_{s} \varphi\left(\frac{x}{N}, \cdot\right)$ we write the difference $N^{2}\left[\varphi\left(\frac{x+X}{N}, \frac{y+Y}{N}\right)-\varphi\left(\frac{x}{N}, \frac{y+Y}{N}\right)\right]$ in the form

$$
N X \varphi_{s}\left(\frac{x}{N}, \frac{y}{N}\right)+\frac{1}{2} \varphi_{s s}\left(\frac{x+\theta X}{N}, \frac{y+Y}{N}\right) X^{2}+\varphi_{s t}\left(\frac{x}{N}, \frac{y+\eta Y}{N}\right) X Y,
$$

where $\theta$ and $\eta$ are random variables taking values from the unit interval. Using the identity $E X=0$, we then find that the contribution from $X \geq-\varepsilon N$ to $K_{N}$ equals

$$
\begin{aligned}
& -N E[X ; X<-\varepsilon N] \varphi_{s}\left(\frac{x}{N}, \frac{y}{N}\right)+E\left[\varphi_{s t}\left(\frac{x}{N}, \frac{y+\eta Y}{N}\right) X Y ; X \geq-\varepsilon N\right] \\
& +E\left[\frac{1}{2} \varphi_{s s}\left(\frac{x+\theta X}{N}, \frac{y+Y}{N}\right) X^{2} ; X \geq-\varepsilon N\right] .
\end{aligned}
$$

It is plain that the first term in (22) is negligible. As for the other two expectations, the contribution from $|Y|>\sqrt{N}$ to them is negligible and in view of (18), $h^{\prime} \leq 0$ and (17) a little inspection shows that the sum of them are dominated by $\frac{1}{2} \sigma_{2}^{2}\left([\pi / 4]^{2}-\frac{1}{6}\right) \varphi_{N}(z)$ if $N$ is large enought and $\varepsilon$ is taken suitably small. Combined with (20) these verify the claimed bound of $-\sigma_{2}^{2} / 16$ if (21) is taken for granted.

Step 3. It remains to verify (21). Observe that $\varphi(s, t) \leq \varphi(s, 0)=C_{0} h\left(s+s_{0}\right)<2 C_{0}$ for all $s, t$ and $\frac{1}{2} C_{0} h\left(s+s_{0}\right) \leq \varphi(s, t)$ whenever $|t| \leq 1$, and then that the quantity on the left side of (21) is dominated by

$$
\frac{2 N^{2}}{h\left(x / N+s_{0}\right)} E\left[h\left(\frac{x+X}{N}+s_{0}\right) ; X<-\varepsilon N\right]+4 N^{2} P[X<-\varepsilon N] .
$$

The second term tends to zero. Denote the first term by $I_{N}$. Since $h(s)<2, I_{N} \rightarrow 0$ uniformly for $0 \leq x \leq 2 \varepsilon N$. For $x>2 \varepsilon N$ we break the expectation involved in $I_{N}$ into two according as $X<-x / 2$ or not and have

$$
I_{N} \leq \frac{2 N^{2}}{h\left(x / N+s_{0}\right)}\left(E\left[h\left(\frac{x+X}{N}+s_{0}\right) ;-x / 2 \leq X<-\varepsilon N\right]+2 P[X<-x / 2]\right) .
$$

Owing to the last property of $u$ in (15) the ratio $h\left(s^{\prime}\right) / h(s)$ is bounded for $1 / 2 \leq s^{\prime} / s<$ $1, s>0$. Hence for some constant $C$,

$$
I_{N} \leq C N^{2} P[X<-\varepsilon N]+C x^{2} e^{u(x / 2)} P[X<-x / 2],
$$

of which the right side approaches zero in view of (16). This completes the proof of (21) and hence that of Lemma 6. 
REMARK 9. If $E\left[X^{2} \log |X|\right]<\infty$, then one can take $u(x)=\log \log (x+e)$ in the proof given above with its considerable simplification and in Lemma 6 the bound $o\left((N / M)^{2}\right)$ can accordingly be replaced by $o(h(M / N))$ with $h(s)=1 / s^{2} \log s$.

3. Proof of Theorem 1. Throughout this section we suppose that $E\left[\tilde{X}^{2} \log |\tilde{X}|\right]<$ $\infty$. We denote by $C, C_{1}, C_{2}$ etc. constants that depend only on the distribution of $(X, Y)$ but whose exact values are irrelevant to our present purpose.

LEMMA 7. Uniformly in $s \in \boldsymbol{Z}$, as $n \wedge k \rightarrow \infty$

$$
G_{L}(i n, s+i k)-G_{D}(i n, s+i k)=o\left(\frac{n k}{s^{2}+(n+k)^{2}}\right) \text {. }
$$

Proof. The difference $G_{L}-G_{D}$ equals $\sum_{y \in D \backslash L} H_{D}(i n, y) G_{L}(y, s+i k)$. Since $G_{L}(i n, s+i k)$ is bounded away from zero if so is $n k /\left(\|s+i(k-n)\|^{2} \vee 1\right)$ (see Theorem 1.1 of [14]) and, in view of Theorem A, $G_{L}(y, s+i k) \leq C G_{L}(y, s-i k)$ (for $y \in D \backslash L$ and $k>0$ ), so that

$$
\sum_{y \in D \backslash L} H_{D}(i n, y) G_{L}(y, s+i k) \leq C \sum_{y \in D \backslash L} H_{D}(i n, y) G_{L}(y, s-i k) \leq C G_{L}(i n, s-i k),
$$

but the last member is $o\left(n k /\left[s^{2}+(n+k)^{2}\right]\right)$ as $n \wedge k \rightarrow \infty$ owing to Theorem A again.

LEMMA 8. For each $n=1,2, \ldots$, uniformly for $|s| \leq k$, as $k \rightarrow \infty$

$$
G_{D}(i n, s+i k)=\frac{2 f_{+}(n) k}{\pi \sigma_{2}^{2}\|s+i k\|^{2}}(1+o(1)) .
$$

PROOF. The proof is similar to that of Lemma 4.1 of [17]. For an integer $N>n$ put $B(N)=\left\{z \in Z^{2} ; \Im z \geq N\right\}$. We employ the representation

$$
G_{D}(i n, s+i k)=E_{i n}\left[G_{D}\left(S_{\tau_{B(N)}}, s+i k\right) ; \tau_{B(N)}<\tau_{D}\right]
$$

in which $G_{D}$ under the expectation may be replaced by $G_{L}$ if $N$ is large enough in view of Lemma 7. For evaluation of the expectation with this replacement being made we apply the following results concerning the one dimensional walk $\Im S_{n}$ that are proved in [17] (Section 2).

LEMma B. Uniformly for $1 \leq n<N$, as $N \rightarrow \infty$

$$
P_{i n}\left[\tau_{B(N)}<\tau_{D}\right]=\frac{f_{+}(n)}{N}+o\left(\frac{n}{N}\right) \text { and } \frac{1}{n} E_{i n}\left[\Im S_{\tau_{B(N)}}-N ; \tau_{B(N)}<\tau_{D}\right] \rightarrow 0 .
$$

In the rest of the proof of Lemma 8, which can be readily adapted for the case when $n / k \rightarrow 0$, we indicate the (natural) dependence on $n$ of the estimates given there, although $n$ is considered to be fixed as in the statement of the lemma.

Theorem A, Lemma 7 and Lemma B together show that for each $\varepsilon>0$ and an integer $n \geq 1$ there exists a positive integer $N>n$ such that for all $s \in \boldsymbol{Z}, k>2 N$ and $N \leq m<k / 2$,

$$
\left|G_{D}(i m, s+i k)-\frac{2 m k}{\pi \sigma_{2}^{2}\|s+i k\|^{2}}\right|<\frac{\varepsilon m k}{s^{2}+k^{2}},
$$


and that

$$
\begin{gathered}
\left|P_{i n}\left[\tau_{B(N)}<\tau_{D}\right]-f_{+}(n) / N\right| \leq \varepsilon n / N, \\
E_{i n}\left[\Im S_{\tau_{B(N)}}-N ; \tau_{B(N)}<\tau_{D}\right] \leq \varepsilon n .
\end{gathered}
$$

If $\tau=\tau_{B(N)} \wedge \tau_{D}$, then $E_{i n}[\tau] \leq C n N$ (which may be easily derived by considering the mean sojourn time spent by $\Im S_{j}$ at each point in the interval $[1, N)$ ), and on using this

$$
\begin{aligned}
P_{i n}\left[\Im S_{\tau_{B(N)}}>k / 2, \tau_{B(N)}<\tau_{D}\right] & =\sum_{j=1}^{\infty} P_{i n}\left[\Im S_{j-1}+Y>k / 2, \tau>j-1\right] \\
& \leq \sum_{j=1}^{\infty} P_{i n}[\tau>j-1] P[Y>k / 2-N] \leq \frac{C n N}{k^{2}}
\end{aligned}
$$

for all $k$ large enough. Since $G_{D}(y, s+i k) \leq C^{\prime} \log k$ (see Remark 1) this entails that

$$
E_{i n}\left[G_{D}\left(S_{\tau_{B(N)}}, s+i k\right) ; \Im S_{\tau_{B(N)}}>k / 2, \tau_{B(N)}<\tau_{D}\right] \leq C^{\prime \prime} n N k^{-2} \log k .
$$

For the other half of the expectation we make decomposition

$$
E_{i n}\left[G_{D}\left(S_{\tau_{B(N)}}, s+i k\right) ; \Im S_{\tau_{B(N)}} \leq k / 2, \tau_{B(N)}<\tau_{D}\right]=I+r_{1}+r_{2}
$$

where, writing $A$ for the event $\left\{\Im S_{\tau_{B(N)}} \leq k / 2, \tau_{B(N)}<\tau_{D}\right\}$,

$$
I=E_{i n}\left[\frac{2 N k}{\pi \sigma_{2}^{2}\left\|s+i k-S_{\tau_{B(N)}}\right\|^{2}} ; A\right], \quad r_{1}=E_{i n}\left[\frac{2\left(\Im S_{\tau_{B(N)}}-N\right) k}{\pi \sigma_{2}^{2}\left\|s+i k-S_{\tau_{B(N)}}\right\|^{2}} ; A\right]
$$

and $r_{2}$ is the rest. Owing to (24) we obtain

$$
\left|r_{2}\right| \leq \varepsilon E_{i n}\left[\frac{\Im S_{\tau_{B(N)}} k}{\pi \sigma_{2}^{2}\left\|s+i k-S_{\tau_{B(N)}}\right\|^{2}} ; A\right] .
$$

From (26) we derive $E_{i n}\left[\Im S_{\tau_{B(N)}} ; \tau_{B(N)}<\tau_{D}\right] \leq N P_{i n}\left[\tau_{B(N)}<\tau_{D}\right]+\varepsilon n \leq C n$; hence $\left|r_{2}\right|<C_{2} \varepsilon n / k$. By (26) again $\left|r_{1}\right| \leq C_{1} \varepsilon n / k$. Choosing $M=M(\varepsilon, n)>1$ so that

$$
P_{i n}\left[\left|S_{\tau_{B(N)}}\right|>M, \tau_{B(N)}<\tau_{D}\right]<\varepsilon n / N,
$$

we may replace $I$ by

$$
E_{i n}\left[\frac{2 N k}{\pi \sigma_{2}^{2}\left\|s+i k-S_{\tau_{B(N)}}\right\|^{2}} ;\left|S_{\tau_{B(N)}}\right| \leq M, \tau_{B(N)}<\tau_{D}\right],
$$

with the error at most $8 \varepsilon / k \pi \sigma_{2}$. In the last expectation, moreover, $S_{\tau_{B(N)}}$ in the denominator of its integrand may be set zero with the error of the order $O\left(1 / k^{2}\right)$ and then, in view of (28) again, the event $\left\{\left|S_{\tau_{B(N)}}\right| \leq M\right\}$ may be deleted. Combined with (27) and (23) this shows that for all sufficiently large $k$,

$$
\left|G_{D}(i n, s+i k)-\frac{2 N k}{\pi \sigma_{2}^{2}\|s+i k\|^{2}} P_{i n}\left[\tau_{B(N)}<\tau_{D}\right]\right|<C_{3} \frac{\varepsilon n}{k}
$$

and substitution from (25) completes the proof of Lemma 8. 
LEMMA 9. For each $n=1,2, \ldots$, uniformly for $k<|s|$, as $|s| \rightarrow \infty$

$$
G_{D}(i n, s+i k)=\frac{2 f_{+}(n) f_{-}(k)}{\pi \sigma_{2}^{2}\|s+i(k-n)\|^{2}}(1+o(1)) .
$$

Proof. First consider the case $k \rightarrow \infty$ under $k \leq|s|$. The proof is the same as the preceding one except for a new ingredient: we need the bound

$$
P_{i n}\left[\left|\Re S_{\tau_{B(N)}}\right|>|s| / 2 ; \tau_{B(N)}<\tau_{D}\right]=o\left(N^{2} / s^{2}\right),
$$

which, however, is readily deduced from Lemma 6 . Employing this bound we obtain as before that

$$
\begin{aligned}
& E_{i n}\left[G_{D}\left(S_{\tau_{B(N)}}, s+i k\right) ; \tau_{B(N)}<\tau_{D}\right] \\
& \quad=E_{i n}\left[\frac{2 N k}{\pi \sigma_{2}^{2}\left\|s+i k-S_{\tau_{B(N)}}\right\|^{2}} ;\left|\Re S_{\tau_{B(N)}}\right| \leq \frac{|s|}{2}, \tau_{B(N)}<\tau_{D}\right]+r
\end{aligned}
$$

with $|r|<C \varepsilon / s^{2}$. We can then proceed as before.

We must still prove the formula of the lemma for each $k=1,2, \ldots$ But by applying what has just been proved as well as Lemma 8 (to the dual walk) we have

$$
\left|G_{D}(i m, s+i k)-\frac{2 m f_{-}(k)}{\pi \sigma_{2}^{2}\|s+i k\|^{2}}\right|<\frac{\varepsilon m k}{s^{2}+k^{2}}
$$

(valid for all $s$ and $m \geq N$ ) in place of (24). The rest is similar (only simpler) to the above.

Combining Lemmas 7 through 9 and taking the duality of roles of $n$ and $k$ into account we conclude the estimate of Theorem 1 .

4. Estimatation of $H_{D}$. Suppose that $E\left[\left|S_{1}^{0}\right|^{2} \log \left|S_{1}^{0}\right|\right]<\infty$ and define the random variable

$$
\boldsymbol{G}=\boldsymbol{G}_{n, s, k}:=G_{D}(i n, s-X+i(k-Y)),
$$

so that (3) is rewritten as

$$
H_{D}(i n, s+i k)=E[\boldsymbol{G} ; Y<k] \quad(n>0, k \leq 0) .
$$

Putting $M=M(s, k, n)=\|s+i(k-n)\|$ and fixing a positive constant $\lambda \leq 1 / 4$ arbitrarily, we split the expectation in (29) into two ones according as $\|X+i Y\|$ is less than $\lambda M$ or not. $\tilde{\boldsymbol{G}}$ by

First consider the case $\|X+i Y\|<\lambda M$. Bringing in the event $\mathcal{A}$ and random variable

$$
\mathcal{A}=\{\|X+i Y\|<\lambda M, Y<k\}, \quad \tilde{\boldsymbol{G}}=\frac{2 f_{+}(n)}{\pi \sigma_{2}^{2}} \cdot \frac{f_{-}(k-Y)}{\|s-X+i(k-Y-n)\|^{2}},
$$

we write

$$
E[\boldsymbol{G} ; \mathcal{A}]=\frac{2 f_{+}(n) E\left[f_{-}(k-Y) ; \mathcal{A}\right]}{\pi \sigma_{2}^{2} M^{2}}+E[\boldsymbol{G}-\tilde{\boldsymbol{G}} ; \mathcal{A}]+r_{1}
$$


where $r_{1}=r_{1}(n, s, k)$ is given by

$$
r_{1}=\frac{2 f_{+}(n)}{\pi \sigma_{2}^{2}} E\left[\frac{f_{-}(k-Y)}{\|s-X+i(k-Y-n)\|^{2}}-\frac{f_{-}(k-Y)}{\|s+i(k-n)\|^{2}} ; \mathcal{A}\right] .
$$

We claim that uniformly for $s \in \boldsymbol{Z}, k \leq 0, n>0$, as $M \rightarrow \infty$

$$
E[|\boldsymbol{G}-\tilde{\boldsymbol{G}}| ; \mathcal{A}]=M^{-2} n q(k) \times o_{b}(1)
$$

with $o_{b}(1)$ being as in Theorem 2. Clearly $(1-\lambda) M \leq\|s-X+i(k-Y-n)\| \leq(1+\lambda) M$ on $\mathcal{A}$ and the boundedness of $o_{b}(1)$ follows from (1) and Theorem 1. Owing to Theorem 1 again, for any $\varepsilon>0$ there exists $\delta>0$ such that for $M>1 / \delta$,

$$
E[|\boldsymbol{G}-\tilde{\boldsymbol{G}}| ; \mathcal{A}, k-Y<\delta M]=\varepsilon M^{-2} n q(k),
$$

while $E[|\boldsymbol{G}-\tilde{\boldsymbol{G}}| ; k-Y \geq \delta M] M^{2} / f_{+}(n) \rightarrow 0$ as $M \rightarrow \infty$ for each $k \leq 0$. Combining these fact verifies the claim (32). Now (30) may be written as

$$
E[\boldsymbol{G} ; \mathcal{A}]=\frac{f_{+}(n) q(k)}{\pi M^{2}}\left(1+o_{b}(1)\right)+r_{1} .
$$

Noting $|\|s-X+i(k-Y-n)\|-M| \leq\|X+i Y\|$ one obtains that

$$
\left|r_{1}\right| \leq \frac{C n}{M^{3}} E\left[f_{-}(k-Y)\|X+i Y\| ; Y<k\right]
$$

and the expectation on the right side tends to zero as $k \rightarrow-\infty$.

Next we evaluate the expectation on $\|X+i Y\| \geq \lambda M$, which we denotet by $r_{2}$ :

$$
r_{2}=r_{2}(n, s, k):=E[G ;\|X+i Y\| \geq \lambda M, Y<k] .
$$

Substituting (33), (29) is accordingly written as

$$
H_{D}(i n, s+i k)=\frac{f_{+}(n) q(k)}{\pi M^{2}}\left(1+o_{b}(1)\right)+r_{1}+r_{2} .
$$

Since $G_{D} \leq C \log (n+1)$,

$$
r_{2} \leq C(\log (n+1)) P[\|X+i Y\| \geq \lambda M, Y<k]
$$

for some constant $C$ and by the assumed moment condition

$$
r_{2}=o\left(\frac{\log (n+1)}{M^{2} \log M}\right) \quad(M=\|s+i(k-n)\| \rightarrow \infty) .
$$

Combined with (34) and (36) this gives (7) for the case $\lambda n>|\tilde{s}|$ as well as (5) with the estimate $r=r_{1}+r_{2}=O\left(\log (n+1) / M^{2} \log M\right)$. Here

$$
\tilde{s}=s-\mu(n-k), \quad \mu=\sigma_{12} / \sigma_{2}^{2} .
$$

It remains to show (7) for $\lambda n \leq|\tilde{s}|$. To this end it suffices to prove that

$$
\frac{s^{2}+n^{2}}{n} \sum_{k<-m} E[\boldsymbol{G} ; Y<k] \rightarrow 0 \quad \text { as } m,|s| \rightarrow \infty
$$


under the constraint $\lambda n \leq|\tilde{s}|$. For evaluation of the expectation above we consider it on each of the following three events, where we write $\tilde{\sigma}_{1}=\sigma^{2} / \sigma_{2}$ so that $\|s+i(k-n)\|^{2}=$ $\left[\sigma_{2}^{2} \tilde{s}^{2}+\tilde{\sigma}_{1}^{2}(n-k)^{2}\right] / \sigma^{2}$; remember $\tilde{X}=X-\mu Y$.

(1) $A_{1}=\{|\tilde{X}|<\lambda|\tilde{s}|\}$ : On this event we have $\boldsymbol{G} \leq \operatorname{Cn}(k-Y) / \tilde{s}^{2}$ if $Y<k$ and the required estimate of $\sum_{k \leq-m} E\left[\boldsymbol{G} ; A_{1}, Y<k\right]$ follows from

(40) $\sum_{k \leq-m} E[k-Y ; Y<k]=\frac{1}{2} E[(-m-Y)(-m-Y+1) ; Y \leq-m] \rightarrow 0$ as $m \rightarrow \infty$.

(2) $A_{2}=\{|k-Y-n|<n$ and $|\tilde{X}| \geq \lambda|\tilde{s}|\}$ : Since $\boldsymbol{G} \leq C \log (n+1)$ (a.s.), the sum $\sum_{k \leq 0} E\left[\boldsymbol{G} ; A_{2}, Y<k\right]$ is dominated by $C \log (n+1)$ times

$$
\begin{aligned}
\sum_{k \leq 0} P\left(A_{2}\right) & \leq \sum_{|l|<n} \sum_{k \leq 0} P[Y=k-n+l,|\tilde{X}| \geq \lambda|\tilde{s}|] \\
& \leq \sum_{|l|<n} P[|\tilde{X}| \geq \lambda|\tilde{s}|]=o\left(\frac{n}{\tilde{s}^{2} \log |\tilde{s}|}\right) .
\end{aligned}
$$

(3) $A_{3}=\{|k-Y-n| \geq n$ and $|\tilde{X}| \geq \lambda|\tilde{s}|\}$ : On $A_{3} \cap\{Y<k\}$ we have $k-Y>2 n$ and, in view of Theorem A and (2), $\boldsymbol{G} \leq C n /|k-Y-n|$ for some constant $C$. Now

$$
\begin{aligned}
\sum_{k \leq 0} E\left[|k-Y-n|^{-1} ; Y<k, A_{3}\right] & \leq E\left[\sum_{Y+2 n<k \leq 0}(k-Y-n)^{-1} ;-Y>2 n,|\tilde{X}| \geq \lambda|\tilde{s}|\right] \\
& \leq E[(1+\log |Y|) ; Y<0,|X| \geq \lambda|\tilde{s}|]=o\left(1 / \tilde{s}^{2}\right) .
\end{aligned}
$$

These prove (39). The proof of Theorem 2 is complete.

5. Estimatation of $G_{L}$ for $n k<0$. For simplicity we let $0<n \leq-k$ unless otherwise stated, which gives rise to no loss of generality in view of duality. The proof is based on the representation

$$
G_{L}(i n, s+i k)=\sum_{m<0} \sum_{j \in Z} H_{D}(i n, j+i m) G_{L}(j+i m, s+i k) \quad(n>0, k<0) .
$$

As in the preceding section put $M=M(j, m, n)=\|j+i(m-n)\|$ to have

$$
H_{D}(i n, j+i m)=\frac{f_{+}(n) q(m)}{\pi M^{2}}(1+o(1))+\left(r_{1}+r_{2}\right)(n, j, m) \quad(m<0)
$$

with $r_{1}$ and $r_{2}$ defined in (31) and (35) with $\lambda=1 / 4$. Suppose $E\left[\left|S_{1}^{0}\right|^{3} \log (|Y| \vee 2)\right]<\infty$. Then we have $\sum_{m<0} q(m)|m| \log |m|<\infty$ and, in place of (34),

$$
r_{1}(n, j, m)=O\left(\frac{n}{M^{4} \log (2-m)}\right) .
$$

We partition the range of summation in (41) into three parts $D_{1}, D_{2}$ and $D_{3}$ defined by

$$
\begin{gathered}
D_{1}=\{k / 2<m<0\}, \quad D_{2}=\left\{|j-s|^{2}+|m-k|^{2}<k^{2} / 4\right\} \\
\text { and } \quad D_{3}=\left\{m \leq k / 2,|j-s|^{2}+|m-k|^{2} \geq k^{2} / 4\right\},
\end{gathered}
$$

and denote the corresponding sums by $I, I I$ and $I I I$, respectively. 
$I$ is the principal part: in view of Theorems A and 2 it may be written as

$$
I=I(s, n, k)=\frac{1}{\pi^{2}} \sum_{k / 2<m<0} \sum_{j \in \boldsymbol{Z}} \frac{f_{+}(n) q(m)}{\|j+i(m-n)\|^{2}} \cdot \frac{\sigma_{2}^{4} a(m) a(-k)+m k}{\sigma_{2}^{2}\|s-j+i(k-m)\|^{2}}(1+o(1)) .
$$

Hence, on using the convolution formula for the Cauchy distribution (cf. Remark 5) with the help of the dominated convergence theorem, as $n \rightarrow \infty$ (under $n \leq-k$ )

$$
I=\frac{1}{\pi}\left(\sum_{m<0} q(m)\left(\sigma_{2}^{2} a(m)+|m|\right)\right) \frac{n-k}{\|s+i(k-n)\|^{2}}(1+o(1)) .
$$

As pointed out in (ii) of Remark 8 we have $\sum_{m<0} q(m)\left(\sigma_{2}^{2} a(m)+|m|\right)=C^{*}-\lambda_{3}$, and the desired formula follows if we show that $I I+I I I=o\left(|k| /\left(s^{2}+k^{2}\right)\right)(n \rightarrow \infty$ under $n<-k)$.

Estimate of II. Change the variables according to $u=j-s$ and $v=m-k$. Then, since $G_{L} \leq C \log \left[k^{2} /\left(u^{2}+v^{2}+1\right)\right]$ on $D_{2}$ (see Theorem 1.1 of [14]),

$$
I I \leq C \sum_{u^{2}+v^{2}<k^{2} / 4}\left[\frac{n q(k+v)}{(s+u)^{2}+(n-v-k)^{2}}+r_{1}+r_{2}\right] \log \frac{k^{2}}{u^{2}+v^{2}+1} .
$$

On $D_{2}$ we have $r_{2}=o\left(\log (n+1) / M^{3} \log |m|\right)=o\left(1 /\left(s^{2}+k^{2}\right)^{3 / 2}\right)$, which combined with

$$
\sum_{u^{2}+v^{2} \leq k^{2} / 4} \log \frac{k^{2}}{u^{2}+v^{2}+1} \leq \sum_{v=-k / 2}^{k / 2} 2 \int_{0}^{k} \log \frac{k^{2}}{u^{2}} d u \leq 4 k^{2},
$$

verifies that the contribution of $r_{2}$ to the sum in (45) is $o\left(k /\left(k^{2}+s^{2}\right)\right)$. The contribution of the other terms are evaluated in a similar way so that $I I=o\left(k /\left(s^{2}+k^{2}\right)\right)$.

Estimate of III. First of all we note that on $\mathrm{D}_{3}$

$$
G_{L}(j+i m, s+i k) \leq \frac{C|m k|}{(s-j)^{2}+m^{2}} .
$$

For the contribution of the main term in (42) to III we first carry out the summation over $j$ with the help of the convolution formula for the Cauchy distribution as before, showing that it is dominated by a constant multiple of

$$
\begin{gathered}
\sum_{m \leq k / 2} \frac{|m| q(m)}{(|m|+n)(|m|+|k|)} \cdot \frac{(2|m|+n+|k|)|m k|}{s^{2}+(2|m|+n+|k|)^{2}} \\
\leq \frac{C k}{s^{2}+k^{2}} \sum_{m \leq k / 2} q(m)|m|=o\left(\frac{k}{s^{2}+k^{2}}\right) .
\end{gathered}
$$

In a similar way the contribution of $r_{1}$ is evaluated with the same bound. That of $r_{2}$ is at most

$$
(\log (n+1)) \sum_{m<k} \sum_{j \in Z} P[\|X+i Y\|>\delta|j|, Y<m] \frac{C|m k|}{(s-j)^{2}+m^{2}},
$$

where $\delta$ is a positive constant depending on $Q$ only. This is $o\left(k /\left(s^{2}+k^{2}\right)\right)$ owing to the following lemma. 
LEMMA 10. If $\alpha$ and $\beta$ are random variables taking values of positive integers, then there exists a universal constant $C$ such that for $k>0, s \geq 0$

$$
\sum_{m=k}^{\infty} \sum_{j=0}^{\infty} P[\alpha>j, \beta>m] \frac{m}{(s-j)^{2}+m^{2}} \leq C \frac{E\left[\alpha^{3}+\beta^{3} ; \beta>k\right]}{s^{2}+k^{2}} .
$$

Proof. Let $J$ be the left side of the formula of the lemma. It may be written as

$$
J=E\left[\sum_{m=k}^{\beta-1} \sum_{j=0}^{\alpha-1} \frac{m}{(s-j)^{2}+m^{2}} ; \beta>k\right] .
$$

If $s \leq k$, we can dominate $J$ by $2 \pi E[\beta ; \beta>k] \leq 2 \pi E\left[\beta^{3} ; \beta>k\right] / k^{2}$ since the inner sum (over $j$ ) is at most $2 \pi$ (for $m$ large enough). Let $s>k$ and break the expectation into two according as $\alpha$ is less or not less than $s / 2$ and name the corresponding ones $E_{1}$ and $E_{2}$. $E_{1}$ is dominated by $s^{-2} E\left[\alpha \beta^{2} ; \beta>k\right]$ as is readily seen. On the other hand $E_{2} \leq 2 \pi E[\beta ; \alpha \geq$ $s / 2, \beta>k] \leq 8 \pi s^{-2} E\left[\alpha^{2} \beta ; \beta>k\right]$.

Thus the proof of Lemma 10, hence of Theorem 4, is complete.

\section{Appendix.}

(A) Minimality of $f_{+}(\Im z)$. In this appendix we prove what is stated in Remark 2 concerning uniqueness of regular function on the upper half plane $\boldsymbol{Z}^{2} \backslash D=\left\{z \in \boldsymbol{Z}^{2} ; \Im z \geq 1\right\}$. We here have only to require that $\Im S_{n}$ is recurrent as a process on $\boldsymbol{Z}$ (no moment condition is assumed). Even under this condition there exists a positive regular function for the walk $\Im S_{n}$ on $[1, \infty)$, which is unique up to a constant factor. We choose any positive regular function and still denote it by $f_{+} \cdot\left(f_{+}(n) / n\right.$ may tend to zero as $n \rightarrow \infty$.)

THEOREM 11. Suppose that $S_{n}$ is irreducible and the one dimensional walk $Y_{n}:=$ $\Im S_{n}$ is recurrent. If $h$ is a regular function on $Z^{2} \backslash D$ for the process killed on $D$ and $h(z) / f_{+}(\Im z)$ is bounded, then $h(z) / f_{+}(\Im z)$ is a constant.

PROOF. It suffices to prove $h(m+1, n)=h(m, n)$ for all $m \in \boldsymbol{Z}$ and $n=1,2, \ldots$ The proof is divided into two steps.

Step 1. We prove that uniformly in $m \in \boldsymbol{Z}$

$$
\lim _{n \rightarrow \infty} \frac{h(m+1, n)-h(m, n)}{f_{+}(n)}=0 .
$$

We adapt the proof given in [11, Theorem 24.1] and reduce the problem to the latter, namely, the fact that any bounded regular function (on $Z^{2}$ ) for $S_{n}$ is constant. Define

$$
s_{n}(m)=h(m, n) / f_{+}(n) \text { and } t(m, n)=s_{n}(m+1)-s_{n}(m)
$$

and put $M=\lim \sup _{n \rightarrow \infty} \sup _{m} t(m, n)$. Owing to the assumed bound of $h s_{n}$ is uniformly bounded, so that we can choose a sequence $(n(k), m(k))$ in $Z^{2}$ such that $n(k) \uparrow \infty$ and for every $(l, j) \in \boldsymbol{Z}^{2}$ there exists

$$
\lim _{k \rightarrow \infty} t(m(k)+l, n(k)+j)=: t^{*}(l, j) \quad \text { with } \quad t^{*}(0,0)=M .
$$


Clearly $t^{*}(l, j) \leq M$. From the hypothesis that $h$ is regular on $Z^{2} \backslash D$ it follows that

$$
\frac{f_{+}\left(n(k)+z_{2}\right) t\left(m(k)+z_{1}, n(k)+z_{2}\right)}{f_{+}(n(k))}
$$

is regular on $z_{2}>-n(k)$ for the process killed on its complement. With the help of Lemma 12 given below we take limit as $k \rightarrow \infty$ and apply the bounded convergence theorem to conclude that $t^{*}(z)$ is regular on $\boldsymbol{Z}^{2}$, hence $t^{*}$ must be identical to $M$. Now for an $\varepsilon>0$ and a positive integer $r$ choose $k$ so that for $l=0,1, \ldots, r-1$,

$$
s_{n(k)}(m(k)+l+1)-s_{n(k)}(m(k)+l) \geq M-\varepsilon,
$$

or, what we are interested in,

$$
s_{n(k)}(m(k)+r)-s_{n(k)}(m(k)) \geq r(M-\varepsilon),
$$

which is possible only if $M \leq 0$ for $s_{n(k)}$ is bounded and non-negative. Thus

$$
\limsup _{n \rightarrow \infty} \sup _{m} t(m, n) \leq 0 .
$$

Arguing with $-t(m, n)$ in place of $t(m, n)$ we have $\liminf _{n \rightarrow \infty} \inf _{m} t_{n}(m) \geq 0$. The proof of (46) is complete.

Step 2. Put $f(m, n)=h(m+1, n)-h(m, n)$; for $N=1,2, \ldots, \tau=\tau_{(-\infty, 0]} \wedge \tau_{[N, \infty)}$ (the first time when $Y_{n}$ exits from $\{1, \ldots, N-1\}$ ); and $M_{n}=f\left(S_{\tau \wedge n}\right)$. It is notified first that

$$
E_{\text {in }}\left[f_{+}\left(Y_{\tau}\right)\right] \leq f_{+}(n),
$$

and then that the martingale $M_{n}$ is uniformly integrable with respect to $P_{z}$ (with $z$ fixed). Now, on using (46) and (47)

$$
|f(z)|=\left|E_{z}\left[f\left(S_{\tau}\right)\right]\right| \leq 2\left(\sup _{j \geq 0} \sup _{m} \frac{f(m, N+j)}{f_{+}(N+j)}\right) E_{z}\left[f_{+}\left(S_{\tau}\right)\right] \rightarrow 0 \quad(N \rightarrow \infty),
$$

showing that $h(z+1)=h(z)$ as desired.

LEMMA 12. $f_{+}$is subadditive, namely $f_{+}(n+k) \leq f_{+}(n)+f_{+}(k)$ for $n, k \geq 1$. (The strict inequality holds if $n+k>2$ and $P[Y \leq-2]>0$.)

Proof. Let $T(k)$ be the first entrance time into $[k, \infty)$ of the strict ascending ladder height process associated with the walk $-\Im S_{n}$. Then $f_{+}(k) / f_{+}(1)=E_{0}[T(k)]-1$ (cf.[11]:Proof of P18.8.; [5], Sections XII.1 and 3) and the Markov property concludes the proof.

(B) An expression of $G_{D}$. Let $t=0,1, \ldots$ denote the time parameter of the walk. Put $\boldsymbol{v}(t, s+i n)=P_{0}\left[S_{t}=s+i n, \tau_{D}>t\right]$ and let $\boldsymbol{v}^{*}$ be its dual (one defined for $-S_{n}$ ). By a duality argument (cf. [5, XII (2.1)])

$$
\boldsymbol{v}^{*}(t, s+i(n-k))=P_{i n}\left[\hat{M}_{t-1}>\hat{M}_{t}=k, \mathfrak{R} S_{t}=s\right],
$$


where $\hat{M}_{t}=\min _{0 \leq t_{1} \leq t} \Im S_{t_{1}}$ and $\hat{M}_{-1}=1$. By making decomposition by means of events analogous to that in the above probability we obtain

$P_{i n}\left[S_{t}=s+i k, \tau_{D}>t\right]=\sum_{t_{1}=0}^{t} \sum_{j=1}^{n \wedge k} \sum_{x=-\infty}^{\infty} \boldsymbol{v}^{*}\left(t_{1}, x+i(n-j)\right) \boldsymbol{v}^{0}\left(t-t_{1}, s-x+i(k-j)\right)$,

where $\boldsymbol{v}^{0}(t, s+i n)=P_{i}\left[S_{t}=s+i(n+1), \tau_{D}>t\right]([1$, Lemma 2.1]). Now taking summation over $t$ we have

$$
G_{D}(i n, s+i k)=\sum_{j=1}^{n \wedge k} \sum_{x=-\infty}^{\infty} \boldsymbol{V}^{*}(x+i(n-j)) \boldsymbol{V}^{0}(s-x+i(k-j)),
$$

where $\boldsymbol{V}^{0}(s+i n)=\sum_{t=0}^{\infty} \boldsymbol{v}^{0}(t, s+i n)=G_{D}(1, s+i(n+1))$ and $\boldsymbol{V}^{*}$ is similarly defined.

It seems hard to derive from the formula (48) an asymptotic form of $G_{D}$ at least in the case when $|s| \rightarrow \infty$ with $n, k$ remaining in a bounded set, even if we know those of $\boldsymbol{V}^{0}$ and $V^{*}$ since the contribution of $x$ around either 0 or $s$ to the sum of (48) is not negligible at all.

Acknowledgments. The author wishes to thank an anonymous referee for his carefully reading the original manuscript and making comments that lead him to improving the readability of the paper.

\section{REFERENCES}

[1] L. Alili And R. A. Doney, Martin boundaries associated with a killed random walk, Ann. Inst. Henri Poincaré Probab. Statist. 37 (2001), 313-338.

[2] P. BIAne, Quantum random walk on the dual of SU(n), Probab. Theory Related Fields 89 (1991), 117-129.

[3] F. CARAVEnna AND L. Chaumont, Invariance principles for random walks conditioned to stay positive, Ann. Inst. Henri Poincaré Probab. Statist. 44 (2008), 170-190.

[4] K. Choquet And J. Deny, Sur l'equation $\mu=\mu * \sigma$, C. R. Acad. Sci. Paris 250 (1960), 799-801.

[ 5 ] W. FELLER, An introduction to probability theory and its applications, Vol. II. Second edition, John Wiley \& Sons, Inc., New York-London-Sydney, 1971.

[6] Y. FukAi AND K. UChiYAma, Potential kernel for two-dimensional random walk, Ann. Probab. 24 (1996), 1979-1992.

[ 7 ] I. Ignatiouk-Robert And C. Loree, Martin boundary of a killed random walk on a quadrant, Ann. Probab. 38 (2010), 1106-1142.

[ 8 ] J. W. Pitman, One-dimensional Brownian motion and the three-dimensional Bessel process, Advances in Appl. Probability. 7 (1975), 511-526.

[ 9 ] K. RASCHEL, Green functions and Martin compactification for killed random walks related to SU(3), Electron. Commun. Probab. 15 (2010), 176-190.

[10] K. RASCHEL, Random walks in the quarter plane absorbed at the boundary: exact and asymptotic, arXiv:0902.2785vl [math.PR].

[11] F. SPItZER, Principles of random walk, The University Series in Higher Mathematics, D. Van Nostrand Co., Inc., Princeton, N.J.-Toronto-London, 1964.

[12] Y. TAMURA AND H. TANAKA, On a formula on the potential operators of absorbing Lévy processes in the half space, Stochastic Process. Appl. 118 (2008), 199-212.

[13] K. UChiYAma, Green's functions for random walks on $\boldsymbol{Z}^{d}$, Proc. London Math. Soc. 77 (1998), 215-240.

[14] K. UChiyama, The Green functions of two dimensional random walks killed on a line and their higher dimensional analogues, Electron. J. Probab. 15 (2010), 1161-1189. 
[15] K. Uchiyama, The hitting distributions of a line for two dimensional random walks, Trans. Amer. Math. Soc. 362 (2010) 2559-2588.

[16] K. Uchiyama, The first hitting time of a single point for random walks, Electron. J. Probab. 16 (2011), 1960-2000.

[17] K. Uchiyama, One dimensional lattice random walks with absorption at a point/on a line, J. Math. Soc. Japan 63 (2011), 675-713.

Department of Mathematics

TOKYO INSTITUTE OF TECHNOLOGY

OH-OKAYAMA, Meguro

TOKYO 152-8551

JAPAN

E-mail address: uchiyama@math.titech.ac.jp 